\title{
EJECTION OF GRAINS FROM COOL STARS
}

\author{
N. C. WICKRA M A S I N G HE
}

Dept. of Applied Mathematics, University College, Cardiff, Wales, U.K.

\begin{abstract}
Dust grains expelled from cool stars are injected into interstellar clouds with typical velocities of $\sim 10^{3} \mathrm{~km} \mathrm{~s}^{-1}$. The interaction of such high-speed grains with gas is discussed. Grains are preferentially stopped within clouds of number density $n_{\mathrm{H}} \approx 10-30 \mathrm{~cm}^{-3}$. Collisions of grains with gas atoms could lead to heating and ionization of clouds and to the production of displacement defects in grains.
\end{abstract}

The process of grain formation in cool stars and the ejection of such grains into interstellar space has been discussed for many years. (Hoyle and Wickramasinghe, 1962; Gilman, 1969). I wish to consider briefly a few consequences of this stellar grain ejection process which have not hitherto been considered. A more detailed discussion is published elsewhere (Wickramasinghe, 1972). To begin with we note that the ratio of radiation pressure to gravity for most grains near a giant star exceeds unity by a large factor. Values of this ratio for various types of spherical particle are set out in Table I for a giant star of $T_{\text {eff }}=3000 \mathrm{~K}, g=1 \mathrm{~cm} \mathrm{~s}^{-2}$ and $R_{*}=3 \times 10^{13} \mathrm{~cm}$

\begin{tabular}{lccc}
\multicolumn{4}{c}{$\begin{array}{c}\text { TABLE I } \\
P / G\end{array}$} \\
\multicolumn{5}{c}{$\left(T_{*}=3000 \mathrm{~K} ; g=1 \mathrm{~cm} \mathrm{~s}^{-2} ; R_{*}=3 \times 10^{13} \mathrm{~cm}\right)$} \\
\hline$a / \mu$ & Iron & Silicate & Graphite \\
\hline 0.01 & $5.59 \times 10^{2}$ & $1.57 \times 10$ & $1.62 \times 10^{3}$ \\
0.03 & $9.18 \times 10^{2}$ & $2.32 \times 10$ & $1.83 \times 10^{3}$ \\
0.05 & $1.70 \times 10^{3}$ & $5.04 \times 10$ & $2.46 \times 10^{3}$ \\
0.08 & $2.84 \times 10^{3}$ & $1.44 \times 10^{2}$ & $3.90 \times 10^{3}$ \\
0.10 & $3.25 \times 10^{3}$ & $2.32 \times 10^{2}$ & $4.72 \times 10^{3}$ \\
0.30 & $1.96 \times 10^{3}$ & $9.55 \times 10^{2}$ & $3.49 \times 10^{3}$ \\
0.50 & $1.02 \times 10^{3}$ & $9.44 \times 10^{2}$ & $1.84 \times 10^{3}$ \\
1.0 & $3.34 \times 10^{2}$ & $4.20 \times 10^{2}$ & $6.24 \times 10^{2}$ \\
\hline
\end{tabular}

Due to these high $P / G$ ratios grains are readily expelled from the regions where they form. Although there would be a strong frictional coupling to the gas near in to the star, grains and gas which expand outwards will quickly decouple. Once grains are driven clear of the photospheric layers they accelerate to very high speeds. The terminal velocity in the absence of an appreciable density of ambient gas is given by

$$
v_{t}=\left[2\left(\frac{P}{G}\right) g R_{0}\right]^{\frac{1}{2}}
$$

where $g$ is the surface gravity and $R_{0} \simeq R_{*}$ is the radial distance at which grain-gas 
decoupling occurs. Ejection velocities of iron, graphite and silicate grains of various radii from a giant star with $T_{*}=3000 \mathrm{~K}, g=1 \mathrm{~cm} \mathrm{~s}^{-2}$ and $R_{*}=3 \times 10^{13} \mathrm{~cm}$ have been calculated and are set out in Table II below.

TABLE II

Ejection velocities in $\mathrm{km} \mathrm{s}^{-1}$; and $\frac{1}{2} m_{\mathrm{H}} V^{2} \mathrm{keV}^{-1}$ within parentheses

\begin{tabular}{llll}
\hline$a / \mu$ & Graphite & Silicate & Iron \\
\hline 0.01 & $3.11 \times 10^{3}(50.66)$ & $3.07 \times 10^{2}(0.49)$ & $1.17 \times 10^{3}(7.21)$ \\
0.03 & $3.31 \times 10^{3}(57.24)$ & $3.73 \times 10^{2}(0.73)$ & $1.45 \times 10^{3}(11.02)$ \\
0.05 & $3.84 \times 10^{3}(77.16)$ & $5.50 \times 10^{2}(1.58)$ & $1.96 \times 10^{3}(20.11)$ \\
0.08 & $4.83 \times 10^{3}(122.04)$ & $9.30 \times 10^{2}(4.51)$ & $2.60 \times 10^{3}(35.24)$ \\
0.10 & $5.32 \times 10^{3}(147.82)$ & $1.18 \times 10^{3}(7.28)$ & $2.86 \times 10^{3}(42.77)$ \\
0.30 & $4.58 \times 10^{3}(109.46)$ & $2.39 \times 10^{3}(29.93)$ & $2.51 \times 10^{3}(32.94)$ \\
0.50 & $3.32 \times 10^{3}(57.60)$ & $2.38 \times 10^{3}(29.57)$ & $1.84 \times 10^{3}(17.59)$ \\
1.00 & $1.94 \times 10^{3}(19.56)$ & $1.59 \times 10^{3}(13.16)$ & $1.08 \times 10^{3}(6.04)$ \\
\hline
\end{tabular}

Grains thus leave their parent stars with typical speeds $10^{3} \mathrm{~km} \mathrm{~s}^{-1}$. The stopping distance of such grains (neglecting disruptive effects) in a cloud of density $n_{H}$ is

$$
\frac{L}{\mathrm{pc}} \cong 3 s\left(\frac{a}{10^{-5} \mathrm{~cm}}\right)\left(\frac{1 \mathrm{~cm}^{-3}}{n_{\mathrm{H}}}\right) \ln \left(\frac{v_{t}}{\mathrm{~km} \mathrm{~s}^{-1}}\right),
$$

where $s$ is the density of grain material. A grain of radius $a \cong 3 \times 10^{-6} \mathrm{~cm}$, density $s \cong 3 \mathrm{~g} \mathrm{~cm}^{-3}$ and initial speed $v_{t} \cong 1000 \mathrm{~km} \mathrm{~s}^{-1}$ is stopped within $\sim 3 \mathrm{pc}$ inside a typical interstellar cloud of number density $n_{\mathrm{H}} \sim 10 \mathrm{~cm}^{-3}$. The stopping distance of the same grain in a tenuous intercloud medium with $n_{\mathrm{H}} \sim \mathrm{cm}^{-3}$ is $\sim 300 \mathrm{pc}$. Since the mean distance between clouds (near the galactic plane) is considerably smaller, $\sim 40 \mathrm{pc}$, this feature would ensure the trapping of grains preferentially within clouds. A fairly natural explanation is thus provided for the observed correlation between $\mathrm{H} \mathrm{I}$ and dust in normal clouds (Lilley, 1955; Heiles, 1967).

The slowing of grains within clouds is attended by several effects. Gas atoms impinging on grains have initial energies $\sim 10^{2} \mathrm{keV}$. Such impacts lead to sputtering of the grains, but it may be shown that their radii are reduced, only by a small factor. A more important effect is the accumulation of solid-state defects. It can be shown that for most types of solid material grains are saturated with a defect concentration of $\sim 1 \%$. Such a defect concentration could produce important optical effects in the grains - e.g. introduce colour centres and increase their infrared absorbtivity.

Finally, I would like to discuss a few implications of the grain slowing process for the interstellar medium itself. A hydrogen atom striking a grain with relative energy $\sim 10^{2} \mathrm{keV}$ is stopped inside it and is eventually re-evaporated at the grain temperature. The re-evaporated atoms/ions have energies $\sim 10^{2} \mathrm{keV}$ and this energy dissipated in the cooler cloud could lead to significant heating and ionization.

The average mass density of grains in a cloud of density $n_{H}$ is

$$
\varrho_{g} \simeq 10^{-2} n_{\mathrm{H}} m_{\mathrm{H}} \mathrm{g} \mathrm{cm}^{-3}
$$


(see, for example, Wickramasinghe, 1967). According to the ideas discussed here a large fraction of this mass density may be assumed to be supplied from cool stars with initial grain speeds corresponding to

$$
\frac{1}{2} m_{\mathrm{H}} v^{2} \simeq 10^{2} \mathrm{keV} \text {. }
$$

The total kinetic energy initially associated with the mass density (3) is

$$
U \simeq \frac{1}{2} \varrho_{g} v^{2} \simeq 1.6 \times 10^{-9} n_{\mathrm{H}} \mathrm{erg} \mathrm{cm}^{-3} .
$$

An appreciable fraction of this energy is converted firstly to kinetic energy of ejected protons, and thence, through their interaction with the gas, into thermal energy of the gas cloud. Assuming the grains are re-supplied with a typical turnover time of $\sim 10^{9} \mathrm{yr}$ we have a heat input from this process given by

$$
\Gamma_{\mathrm{gr}} \simeq 5 \times 10^{-26} n_{\mathrm{H}} \mathrm{erg} \mathrm{cm}^{-3} \mathrm{~s}^{-1} \text {. }
$$

The associated ionization rate is obtained by dividing (6) by $\sim 30 \mathrm{eV}$ corresponding to each ionization (the kinetic energy liberated by primary collisions of protons of energy $\sim 100 \mathrm{keV}$ with $\mathrm{H}$ atoms (Bethe, 1933)). Thus we have an ionization rate

$$
\zeta_{\mathrm{gr}} \simeq 10^{-15} \mathrm{n}_{\mathrm{H}} \mathrm{cm}^{-3} \mathrm{~s}^{-1} \text {. }
$$

The above values of $\Gamma$ and $\zeta$ exceed those derived from earlier arguments of subcosmic ray heating (Hayakawa et al., 1961 ; Spitzer and Tomasko, 1968; Spitzer and Scott, 1969) and of X-ray heating (Silk and Werner, 1969) by a factor $\sim 10$. Allowing even for an efficiency factor as low as 0.1 it is clear that the present process could well provide the dominant source of heating and ionization of interstellar gas.

\section{References}

Bethe, H.: 1933, Handbuch der Physik 24, 491.

Gilman, R. C.: 1969, Astrophys. J. 155, L185.

Heiles, C.: 1967, Astrophys. J. 148, 299.

Hayakawa, S., Nishimura, S., and Takayanagi, K.: 1961, Publ. Astron. Soc. Japan 13, 184.

Hoyle, F. and Wickramasinghe, N. C.: 1962, Monthly Notices Roy. Astron. Soc. 124, 417.

Lilley, A. E.: 1955, Astrophys. J. 121, 559.

Silk, J. and Werner, M. W.: 1969, Astrophys. J. 158, 185.

Spitzer, L. and Scott, E. H.: 1969, Astrophys. J. 158, 161.

Spitzer, L. and Tomasko, M. G.: 1968, Astrophys. J. 152, 971.

Wickramasinghe, N. C.: 1967, Interstellar Grains, Chapman \& Hall Co., London.

Wickramasinghe, N. C.: 1972, Monthly Notices Roy. Astron. Soc. 159, 269. 